\title{
Simultaneous Determination of Catalpol, Aucubin, and Geniposidic Acid in Different Developmental Stages of Rehmannia glutinosa Leaves by High Performance Liquid Chromatography
}

\author{
Yanjie Wang, ${ }^{1,2}$ Dengqun Liao, ${ }^{1}$ Minjian Qin, ${ }^{2}$ and Xian'en $\mathrm{Li}^{1}$ \\ ${ }^{1}$ Institute of Medicinal Plant Development, Chinese Academy of Medical Sciences and Peking Union Medical College, \\ Beijing 100193, China \\ ${ }^{2}$ Department of Resources Science of Traditional Chinese Medicines, China Pharmaceutical University, Nanjing 210009, China \\ Correspondence should be addressed to Xian’en Li; xianenli@yeah.net
}

Received 29 March 2016; Revised 15 May 2016; Accepted 8 June 2016

Academic Editor: Giuseppe Ruberto

Copyright (C) 2016 Yanjie Wang et al. This is an open access article distributed under the Creative Commons Attribution License, which permits unrestricted use, distribution, and reproduction in any medium, provided the original work is properly cited.

\begin{abstract}
Although $R$. glutinosa roots are currently the only organ source in clinics, its leaves are a potential supplement for the roots especially in extraction of some important bioactive compounds. Our early work found that the contents of catalpol and total iridoid glycosides varied among different developmental stages of R. glutinosa leaves. Aucubin and geniposidic acid, the abundant major bioactive compounds in Eucommia ulmoides and Gardenia jasminoides, respectively, were found present in R. glutinosa roots, however, and have not been analyzed in its leaves. In this paper, we aimed to determine contents of these three iridoid glycosides in different developmental stages of $R$. glutinosa leaves using the optimized HPLC-UV conditions. Our results showed that aucubin and GPA in R. glutinosa leaves were much lower than catalpol and showed the increasing trend with the leaf development, which was different from catalpol. This work provided the important information for future exploitation of R. glutinosa leaves as a potential supplement for its roots in extraction of some important bioactive compounds and studying the relationship of aucubin and catalpol metabolism.
\end{abstract}

\section{Introduction}

Rehmannia glutinosa Libosch (dihuang in Chinese) belongs to the family Scrophulariaceae. Its raw or processed roots are the only organ source for clinical uses of traditional Chinese medicine, and the leaves are not utilized at all. However, R. glutinosa leaves have the pharmacological effects and can nourish yin, tonify qi and kidney, and promote blood circulation [1]. In the folk, the fresh leaves are often applied externally to treat malignant sore and tinea manus and pedis [2]. As a perennial herb, R. glutinosa has to be annually cultivated and harvested since its fleshy roots easily decay during the winter dormancy or are consumed when new plantlets come out from them in the next season. Due to its severe continuous cropping obstacle, however, $R$. glutinosa plants could not grow well on the same land after the first cropping, decreasing the root yield greatly [3]. Thus, exploitation of $R$. glutinosa leaves not only provides another potential medicinal source for extraction of important bioactive compounds present in its roots but also complements the shortage of R. glutinosa roots due to the limited land and continuous cropping obstacle.

A lot of pharmacologically bioactive secondary metabolites $[4,5]$ including iridoid glycosides, phenylethanoid glycosides, and polysaccharides have been isolated in R. glutinosa, of which iridoid glycosides are thought to be main bioactive constituents. Till now, more than thirty iridoid glycosides have been separated and identified [4-6], including catalpol, aucubin, geniposidic acid (GPA), rehmanniosides A, B, C, and $\mathrm{D}$, and rehmaglutosides $\mathrm{A}-\mathrm{K}$. The pharmacological effects of some of these iridoid glycosides have been extensively studied. For example, as the main active iridoid glycoside in R. glutinosa, catalpol was found to play the important roles in treatment of many diseases including kidney diseases 
[7], neurodegenerative diseases [8], and diabetes $[9,10]$. It is revealed that aucubin had pharmacological effects such as antifungals [11], anti-inflammation and antioxidation [12], and hepatoprotection [13]. Similarly, GPA had therapeutic effects in anti-inflammation, liver disorders, and antinociception $[14,15]$. Among these three compounds, only catalpol was assayed in detail to reveal its spatiotemporal expression profiling among different developmental stages of $R$. glutinosa leaves and between leaf and root $[16,17]$. It is shown that catalpol content varied among different developmental stages of $R$. glutinosa leaves and within the whole growth stage. Aucubin and GPA were found to accumulate in roots of $R$. glutinosa $[18,19]$, and only the former was quantified and much lower than catalpol in the root $[18,20]$. Therefore, in this paper, we aimed to determine the content of these three iridoid glycosides of two $R$. glutinosa cultivars in different developmental stages of leaves using HPLC-UV method and compared their changing trend.

\section{Materials and Methods}

2.1. Plant Material. Two primary cultivars of R. glutinosa, Wen 85-5 and Beijing no. 1, were grown in around midApril in 2014 at the IMPLAD (Institute of Medicinal Plant Development), Beijing, China. The plant interval was $40 \mathrm{~cm}$ $\times 40 \mathrm{~cm}$. Normal field management was applied during the growth period. On 11th September, 2014, the top eight positions of leaves from each plant were collected separately, washed to remove the surface soil, and then stored at $-80^{\circ} \mathrm{C}$ till use.

2.2. Reagents and Standard Solution Preparation. HPLC grade acetonitrile (ACN) and formic acid were purchased from Thermo Fisher (USA). The standard catalpol $(\geq 98 \%)$ was obtained from the National Institute for Food and Drug Control, China (http://www.nifdc.org.cn/). Standards of aucubin (GR-133-140104) and geniposidic acid (GR-133140423) were purchased from Nanjing Guangrun Biotechnology Co. Ltd., China (http://www.grbiology.com/). The purified water from Wahaha was used throughout the experiment. Other reagents of analytical grade were from Beijing Chemical Industry Inc., China.

The concentrations of catalpol, aucubin, and geniposidic acid in the mixed working stock standard solution were, separately, $0.5025 \mathrm{mg}, 0.0375 \mathrm{mg}$, and $0.0020 \mathrm{mg}$ in $1 \mathrm{~mL}$ of purified water. To make calibration curves, six series concentrations, ranging from $0.01570 \sim 0.5025 \mathrm{mg} / \mathrm{mL}$ for CA, $0.001172 \sim$ $0.0375 \mathrm{mg} / \mathrm{mL}$ for $\mathrm{AU}$, and $6.281 E-05 \sim 0.002010 \mathrm{mg} / \mathrm{mL}$ for GPA, were then separately prepared by diluting the stock working solution with twofold difference. Calibration curves were constructed by plotting the logarithm of the HPLCUV peak areas versus the logarithm concentration of each standard.

2.3. Extraction and Assay of Three Iridoid Glycosides in $R$. glutinosa Leaves. The frozen samples were homogenized with liquid nitrogen and one gram of each sample powder was extracted twice with $25 \mathrm{~mL}$ of $30 \%$ methanol in an ultrasonic water bath at $25^{\circ} \mathrm{C}$ for $20 \mathrm{~min}$. The extract was centrifuged at $12000 \mathrm{rpm}$ for $5 \mathrm{~min}$. The supernatants from two cycles of extraction were combined and evaporated to dryness in a rotary evaporator at $50^{\circ} \mathrm{C}$ under the reduced pressure, redissolved in $50 \mathrm{~mL}$ of pure water, and then filtered with a $0.22 \mu \mathrm{m}$ Millipore membrane filter prior to HPLC analysis.

For HPLC-UV quantification, ten microliters of filtered extracts and standards was run at $30^{\circ} \mathrm{C}$ on a waters $600 \mathrm{E}$ system equipped with a Phenomenex Kinetex C18 column (4.6 mm $\times 100 \mathrm{~mm}, 2.6 \mu \mathrm{m})$, waters 2487 dual wavelength detector, and 2707 autosampler (USA). The isocratic mobile phase contained acetonitrile (5\%) and $0.1 \%$ formic acid in water $(95 \%)$ and was delivered at $0.4 \mathrm{~mL} / \mathrm{min}$. Catalpol and aucubin were monitored at $\lambda 210 \mathrm{~nm}$ and geniposidic acid was monitored at $\lambda 240 \mathrm{~nm}$. The presence of the three iridoid glycosides in $R$. glutinosa leaves was determined by comparing both retention time and spectral data with those of their corresponding authentic standards. Concentrations of three iridoid glycosides in the samples were determined from the below linear standard calibration curves. Their content was shown in dry weight. Since catalpol and other iridoids are not heat-stable, the batch of leaf samples was used to calculate the dry weight of each stage leaf. Three replicates of each stage of leaf sample were measured.

\section{Results and Discussion}

3.1.Optimization of HPLC-UV Conditions. HPLC-UV method has been successfully applied to quantify catalpol (CA), aucubin (AU), and GPA in medicinal plants $[16,17,20-$ 22]. The HPLC conditions that researchers used varied in terms of the columns, mobile phase system, and detection wavelengths. Therefore, we optimized HPLC-UV conditions in terms of column type, UV wavelength, mobile phase composition, addition of formic acid in aqueous phase, and ratio of ACN and water in our early work (Table 1). Several UV wavelengths (e.g., $203 \mathrm{~nm}, 205 \mathrm{~nm}, 206 \mathrm{~nm}$, and $210 \mathrm{~nm}$ ) were used to detect the presence and content of catalpol and (or) aucubin. We found that there was no significant difference in detecting these two iridoid glycosides under 203 210 nm and thus chose $210 \mathrm{~nm}$ as the detection wavelength, which was used in China pharmacopoeia (2010). There was no much difference of the wavelengths $(230 \sim 240 \mathrm{~nm})$ in peak shape and separation of GPA except that less miscellaneous peaks and stable baselines were produced at $\lambda 240 \mathrm{~nm}$. Thus, GPA was detected at $\lambda 240 \mathrm{~nm}$ in latter experiments. As Ji et al. [16] revealed, adding or not adding formic acid (FA) in the mobile phase did not obviously influence the peak shape and separation of catalpol and aucubin, except for GPA. While investigating the difference of different concentrations of ACN in the mobile phase system on the chromatogram, we found that the elution times of the analytes decreased with ACN concentration increased (Table 2). When ACN was higher than $5 \%$, catalpol peak was overlapped with the solvent peak. However, when less than $5 \%$ of ACN was used in the mobile phase, more late AU and GPA were eluted. All the three analytes can be eluted within 10 minutes under $5 \%$ of ACN. In order to shorten the running time for compounds behind them, we adopted $5 \%$ of $\mathrm{ACN}$ in the final mobile phase system. The final HPLC conditions that we adopted to 
TABLE 1: Optimization of HPLC-UV conditions for three analytes.

\begin{tabular}{|c|c|c|c|}
\hline Chromatographic factor & Compared parameter & Optimized conditions & Main advantages \\
\hline Column & $\begin{array}{c}\text { Phenomenex Hydro_RP }(4.6 \mathrm{~mm} \times \\
250 \mathrm{~mm}, 4 \mu \mathrm{m}), \text { Kinetex-C18 } \\
(4.6 \mathrm{~mm} \times 100 \mathrm{~mm}, 2.6 \mu \mathrm{m})\end{array}$ & Kinetex-C18 & $\begin{array}{l}\text { Improved column efficiency, speed, } \\
\text { separation, and sensitivity }\end{array}$ \\
\hline$\lambda_{\mathrm{UV}}$ & $\begin{array}{l}203 \sim 210 \mathrm{~nm} \text { for CA and } \mathrm{AU}, \\
235 \sim 240 \mathrm{~nm} \text { for GPA }\end{array}$ & $\begin{array}{l}210 \mathrm{~nm} \text { for CA and } \\
\mathrm{AU} ; 240 \mathrm{~nm} \text { for GPA }\end{array}$ & $\begin{array}{c}\text { No difference in } 203 \sim 210 \mathrm{~nm} \text {; less } \\
\text { miscellaneous peaks and stabler baseline at } \\
\lambda 240 \mathrm{~nm}\end{array}$ \\
\hline \multirow{3}{*}{ Mobile phase } & Methanol- $\mathrm{H}_{2} \mathrm{O}, \mathrm{ACN}-\mathrm{H}_{2} \mathrm{O}$ & $\mathrm{ACN}-\mathrm{H}_{2} \mathrm{O}$ & $\begin{array}{c}\text { Better peak shape and separation, stabler } \\
\text { baseline }\end{array}$ \\
\hline & $\begin{array}{l}\text { Adding or not adding of formic acid in } \\
\qquad \mathrm{H}_{2} \mathrm{O}\end{array}$ & (0.1\% formic acid) & $\begin{array}{l}\text { Better peak shape and separation for GPA; } \\
\text { no obvious influence on CA and AU }\end{array}$ \\
\hline & Linear gradient or isocratic & Isocratic & $\begin{array}{c}\text { Smooth baseline, better separation, less } \\
\text { miscellaneous peaks }\end{array}$ \\
\hline $\mathrm{ACN}: \mathrm{H}_{2} \mathrm{O}(0.1 \% \mathrm{FA})$ & $1: 99,2: 98,3: 97,5: 95,7: 93,10: 90$ & $5: 95$ & $\begin{array}{c}\text { Shorter elution time; however, CA } \\
\text { overlapped with solvent peak when ACN } \\
\text { was }>5 \% \text {; longer elution time for AU and } \\
\text { GPA when ACN was less than } 5 \%\end{array}$ \\
\hline
\end{tabular}

TABLE 2: Elution time of three analytes under different concentrations of ACN.

\begin{tabular}{lccc}
\hline$\%$ of ACN & Rt $(\min )$ of CA & Rt $(\min )$ of AU & Rt (min) of GPA \\
\hline $1 \%$ & 13.651 & 22.131 & 31.184 \\
$2 \%$ & 7.898 & 11.408 & 23.006 \\
$3 \%$ & 4.361 & 7.023 & 14.070 \\
$5 \%$ & 3.436 & 4.941 & 9.943 \\
$7 \%$ & $\leq$ Rt0 & 3.427 & 8.677 \\
$10 \%$ & $\leq$ Rt0 & $\leq$ Rt0 & 6.181 \\
\hline
\end{tabular}

Note: Rt0 represents solvent peak, about 3.13 3.17.

determine CA, AU, and GPA in our study were summarized in Table 1.

Using the optimized conditions, we further tested our HPLC-UV conditions by checking its linearity, precision, stability, and recovery (Table 3 ). Figure 1 showed that standards of CA, AU, and GPA were eluted separately at $3.412 \mathrm{~min}$, $4.857 \mathrm{~min}$, and $9.850 \mathrm{~min}$. Their corresponding compounds in the sample extract were detected at $3.436 \mathrm{~min}, 4.941 \mathrm{~min}$, and $9.813 \mathrm{~min}$, respectively. The linear regression equations obtained were as follows: CA, $y=0.9707 x+6.7723, R^{2}=$ 0.9997; $\mathrm{AU}, y=0.9952 x+6.8458, R^{2}=0.9999$; and GPA, $y=$ $1.0136 x+7.5797, R^{2}=0.9996$. For the precision estimation, a sample extract was assayed successively for 6 times. The precisions were evaluated as the relative standard deviation (\%, RSD) and, calculated for CA, AU, and PGA, were $0.14 \%, 2.65 \%$, and $2.05 \%$, respectively. This indicated that our instrument was in good precision condition. Repeatability was obtained via parallel preparation of six sample extracts from the same batch. RSD for CA, AU, and PGA was 0. $25 \%, 3.08 \%$, and $2.41 \%$, respectively, which indicated that our extraction method was reproducible. To check the stability of extracts, the same sample extract was assayed separately at $0,2,4,8,12,16$, and $24 \mathrm{~h}$ after extraction. The RSD values for $\mathrm{CA}, \mathrm{AU}$, and PGA were $1.46 \%, 2.38 \%$, and $0.43 \%$, respectively, indicating that our sample extract remained stable at least till 24 hours. The extraction recovery was determined by comparing the content of the compound extracted from the samples with the content of compound from nonextracted standard solutions at equivalent concentrations. To test the sample extraction recovery, one $\mathrm{mL}$ of the standard solution containing $4.824 \mathrm{mg}$ of CA, $0.225 \mathrm{mg}$ of $\mathrm{AU}$, and $0.0134 \mathrm{mg}$ of GPA, respectively, was added into one gram of leaf powder at the beginning of the extraction. The recovery rates for standards CA, AU, and PGA were 102.59\%, 98.29\%, and $101.96 \%$, respectively. The calculated LOD (limit of detection) at $\mathrm{S} / \mathrm{N}>3$ was, separately, $1.3333 E-04 \mathrm{mg} / \mathrm{mL}$ for CA, $1.8170 E-04 \mathrm{mg} / \mathrm{mL}$ for $\mathrm{AU}$, and $4.9926 E-05 \mathrm{mg} / \mathrm{mL}$ for GPA.

3.2. The Content of Catalpol, Aucubin, and GPA Changed with the Leaf Development. R. glutinosa is a perennial herb with cespitose leaves on its shortened stem. The leaf biomass of $R$. glutinosa cultivars increased greatly from the seedling stage till 120 DAP (days after plantation) and then deceased after mid-September (140 DAP), partially due to ongoing senescence of bottom leaves (Figure 2). However, the top eight positions of leaves still stayed green and varied in leaf size, which can represent the different developmental stages of leaves (Figure 3). The 8th leaves, counted downwards from the uppermost leaves, looked similarly in their size and appearance status as the lower positions of unsenescent leaves. However, their dry weight (dry weight per gram fresh weight) changed dynamically with leaf development (Figure 4). Overall, leaf dry weight decreased with the leaf development and increased for L5 and then decreased in the rest of older leaves. L1-L8 had about 0.14-0.18 grams of dry biomass per gram fresh biomass. In this paper, we just focused on the contents of CA, AU, and GPA in the top eight positions of leaves and investigated their relationship with the leaf developmental stages. Although the contents of CA, $\mathrm{AU}$, and GPA were somehow different between Wen 85-5 and Beijing No. 1, the changing trends of these three iridoid glycosides with the leaf developmental stage were very similar 
TABLE 3: Validation of the optimized HPLC-UV conditions for three analytes.

\begin{tabular}{lccccccc}
\hline Iridoid & Calibration curve & $\begin{array}{c}\text { Correlation } \\
\left(R^{2}\right)\end{array}$ & $\begin{array}{c}\text { Precision } \\
(\mathrm{RSD} \%)\end{array}$ & $\begin{array}{c}\text { Reproducibility } \\
(\mathrm{RSD})\end{array}$ & $\begin{array}{c}\text { Stability } \\
(\mathrm{RSD} \%)\end{array}$ & $\begin{array}{c}\text { Recovery } \\
(\%)\end{array}$ & $\begin{array}{c}\text { LLOD } \\
(\mathrm{mg} / \mathrm{mL})\end{array}$ \\
\hline Catalpol & $y=0.9707 x+6.7723$ & 0.9997 & 0.14 & 0.25 & 1.46 & 102.59 & $1.3333 E-04$ \\
Aucubin & $y=0.9952 x+6.8458$ & 0.9999 & 2.65 & 3.08 & 2.38 & 98.29 & $1.8170 E-04$ \\
GPA & $y=1.0136 x+7.5797$ & 0.9996 & 2.05 & 2.41 & 0.43 & 101.96 & $4.9926 E-05$ \\
\hline
\end{tabular}

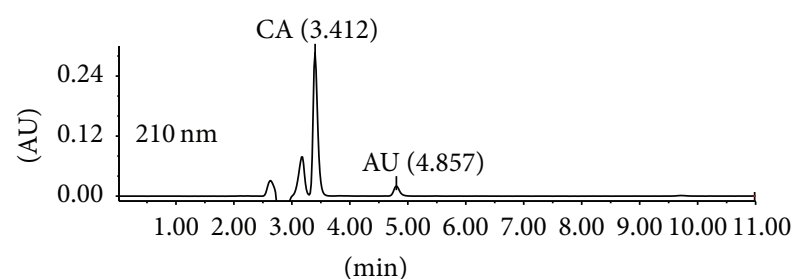

(a)

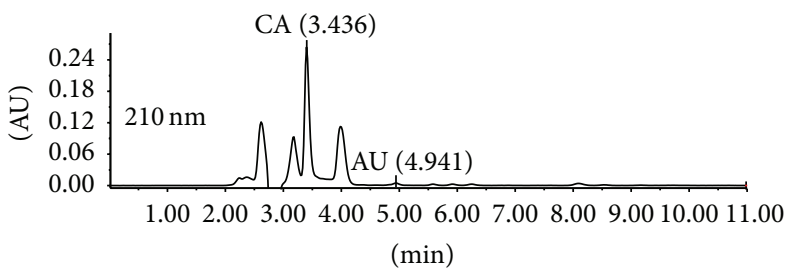

(c)

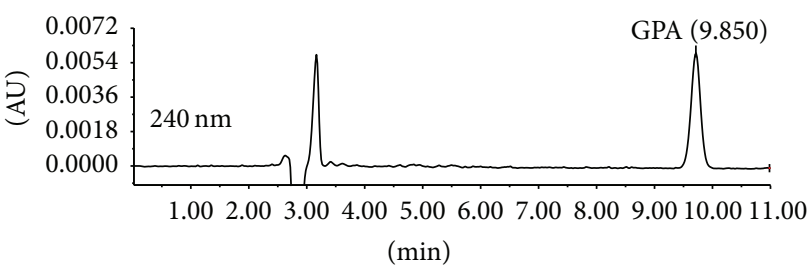

(b)

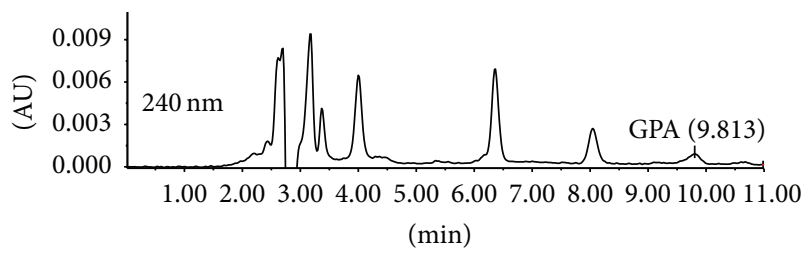

(d)

FigURE 1: HPLC-UV chromatographs of standard mixture ((a), (b)) and R. glutinosa extract ((c), (d)).

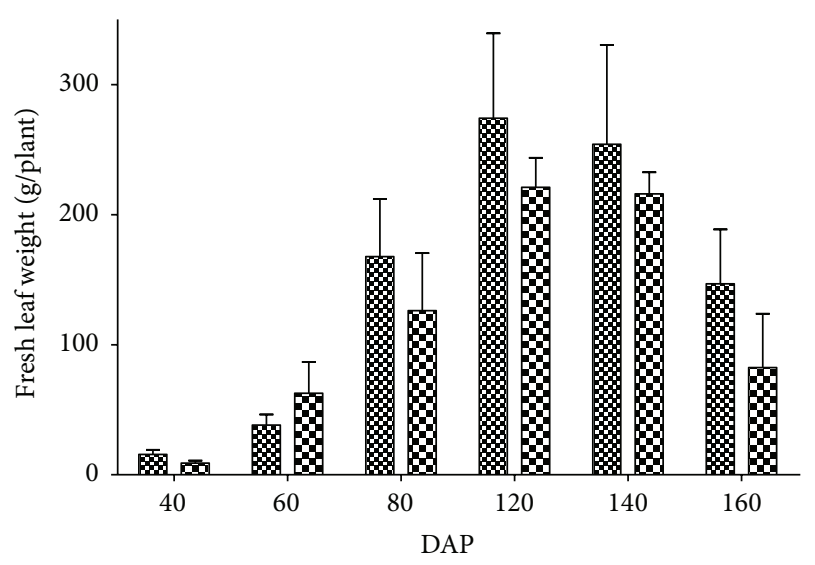

W Wen $85-5$

Beijing No. 1

FIGURE 2: Changes of fresh weight of R. glutinosa leaves during the growth period.

between the two cultivars, indicating no genetic difference in developmental regulation of these three iridoid glycosides (Figures 5 and 6). Generally, catalpol, a major bioactive iridoid glycoside of $R$. glutinosa, was higher in younger leaves and decreased with the leaf development, which was consistent with the result of Ji et al. [16]. Likewise in $R$. glutinosa root [18], aucubin in the leaf was still much lower than catalpol, which was found by Piątczak et al. [23]. The

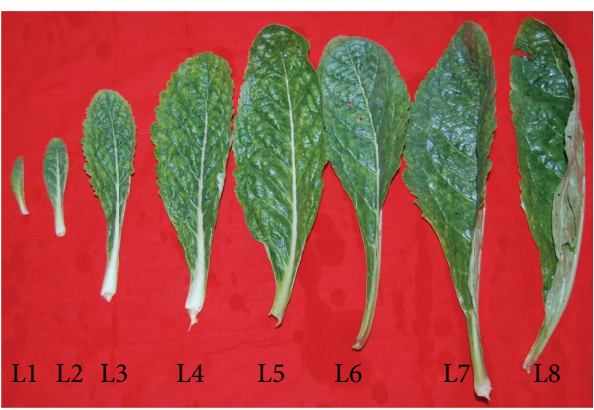

FIgURE 3: Leaf samples of $R$. glutinosa used in the study. L1-L8 represented leaves located on the top position of the stem and downwards.

changing trend of aucubin among different developmental stages of $R$. glutinosa leaves was distinct from catalpol; that is, the older the leaves, the higher the aucubin in them. It was found that aucubin was one of the intermediates for catalpol biosynthesis in Scutellaria albida and Paulownia tomentosa [24]. The opposite metabolic profiling of aucubin and catalpol in $R$. glutinosa leaf indicated that catalpol might not be synthesized via aucubin; however, this needs to be further investigated. GPA was detected to be present in $R$. glutinosa leaves and the lowest analyte in all the developmental stages of $R$. glutinosa leaves. Like aucubin, GPA was increased with the leaf development and higher in older leaves. 


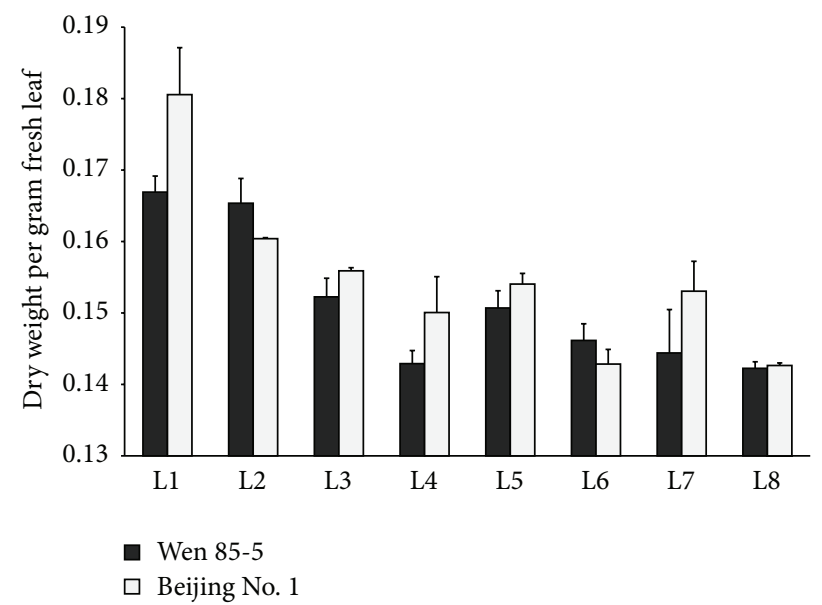

FIGURE 4: Dry weight of R. glutinosa L1-L8 leaves.

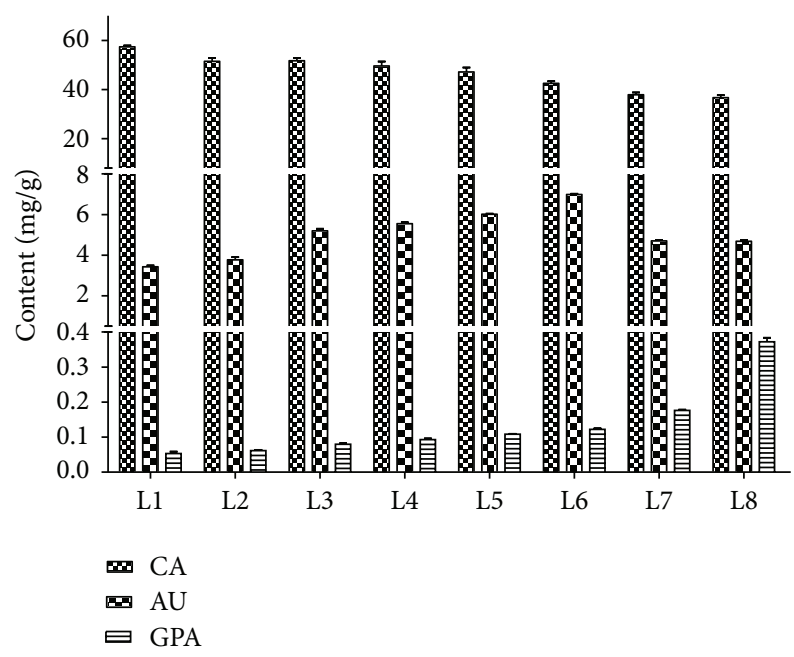

FIGURE 5: Content of catalpol, aucubin, and geniposidic acid in different developmental stages of Wen 85-5 leaves.

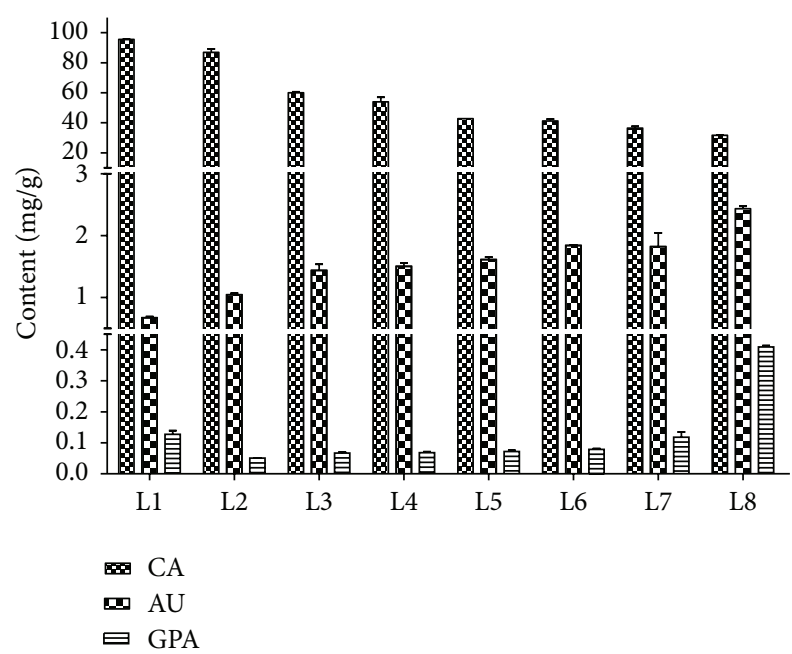

FIGURE 6: Content of catalpol, aucubin, and geniposidic acid in different developmental stages of Beijing No. 1 leaves.

\section{Conclusion}

In this paper, we determined contents of catalpol, aucubin, and geniposidic acid in different developmental stages of $R$. glutinosa leaves using the optimized HPLC-UV conditions and further compared their changing trend with the leaf development. Our results showed that aucubin and GPA in $R$. glutinosa leaves were much lower than catalpol and had the increasing trend with the leaf development, which was different from catalpol. This work provided an important basis for future exploitation of R. glutinosa leaves such as isolation of interesting metabolites, for example, the high concentration compound present at 4.16, which was further identified by HPLC-LC-MS method. It also provided a model to study the relationship of aucubin and catalpol metabolisms in R. glutinosa.

\section{Competing Interests}

The authors declare that there are no competing interests.

\section{Authors' Contributions}

Yanjie Wang and Dengqun Liao contributed equally to this work.

\section{Acknowledgments}

This work was supported by the National Natural Science Foundation of China (81274014).

\section{References}

[1] Beijing Municipal Health Bureau, Standards for Chinese Medicinal Herbs in Beijing, Capital Normal University Press, Beijing, China, 1998.

[2] Nanjing University of Chinese Medicine, Dictionary of Traditional Chinese Medicine, vol. 1, Shanghai Scientific \& Technical, Shanghai, China, 2006.

[3] D. Q. Liao, X. C. Pang, S. Sun, J. J. Qi, L. L. Zhou, and X. E. Li, "Identification of Rehemmina glutinosa germplasm tolerant to continuous cropping and their heritabililty," Crops, vol. 6, pp. 54-58, 2015.

[4] R.-X. Zhang, M.-X. Li, and Z.-P. Jia, "Rehmannia glutinosa: review of botany, chemistry and pharmacology," Journal of Ethnopharmacology, vol. 117, no. 2, pp. 199-214, 2008.

[5] H. W. Li and X. L. Meng, "Research progress on chemical constituents and pharmacological activities of Rehmannia glutinosa," Drug Evaluation Research, vol. 38, pp. 218-228, 2015.

[6] Y.-F. Liu, D. Liang, H. Luo et al., "Hepatoprotective iridoid glycosides from the roots of Rehmannia glutinosa," Journal of Natural Products, vol. 75, no. 9, pp. 1625-1631, 2012.

[7] M. Zhao, J. Tao, D. Qian et al., "Simultaneous determination of loganin, morroniside, catalpol and acteoside in normal and chronic kidney disease rat plasma by UPLC-MS for investigating the pharmacokinetics of Rehmannia glutinosa and Cornus officinalis Sieb drug pair extract," Journal of Chromatography B: Analytical Technologies in the Biomedical and Life Sciences, vol. 1009-1010, pp. 122-129, 2016. 
[8] B. Jiang, R. F. Shen, J. Bi, X. S. Tian, T. Hinchliffe, and Y. Xia, "Catalpol: a potential therapeutic for neurodegenerative diseases," Current Medicinal Chemistry, vol. 22, no. 10, pp. 12781291, 2015.

[9] J. Zhou, G. Xu, S. Ma et al., "Catalpol ameliorates high-fat dietinduced insulin resistance and adipose tissue inflammation by suppressing the JNK and NF- $\kappa \mathrm{B}$ pathways," Biochemical and Biophysical Research Communications, vol. 467, no. 4, pp. 853858, 2015.

[10] J.-P. Shieh, K.-C. Cheng, H.-H. Chung, Y.-F. Kerh, C.-H. Yeh, and J.-T. Cheng, "Plasma glucose lowering mechanisms of catalpol, an active principle from roots of rehmannia glutinosa, in streptozotocin-induced diabetic rats," Journal of Agricultural and Food Chemistry, vol. 59, no. 8, pp. 3747-3753, 2011.

[11] K. P. Shirley, L. J. Windsor, G. J. Eckert, and R. L. Gregory, "In vitro effects of Plantago major extract, aucubin, and baicalein on Candida albicans biofilm formation, metabolic activity, and cell surface hydrophobicity," Journal of Prosthodontics, 2015.

[12] E. Reina, N. Al-Shibani, E. Allam, K. S. Gregson, M. Kowolik, and L. J. Windsor, "The effects of Plantago major on the activation of the neutrophil respiratory burst," Journal of Traditional and Complementary Medicine, vol. 3, no. 4, pp. 268-272, 2013.

[13] I.-M. Chang, "Liver-protective activities of aucubin derived from traditional oriental medicine," Research Communications in Molecular Pathology and Pharmacology, vol. 102, no. 2, pp. 189-204, 1998.

[14] H. Chen, X. Huang, J. Min et al., "Geniposidic acid protected against ANIT-induced hepatotoxity and acute intrahepatic cholestasis, due to Fxr-mediated regulation of Bsep and Mrp2," Journal of Ethnopharmacology, vol. 179, pp. 197-207, 2016.

[15] N. Gong, H. Fan, A.-N. Ma, Q. Xiao, and Y.-X. Wang, "Geniposide and its iridoid analogs exhibit antinociception by acting at the spinal GLP-1 receptors," Neuropharmacology, vol. 84, pp. 31-45, 2014.

[16] X.-Q. Ji, P. Sun, J.-J. Qi, D.-Q. Liao, and X.-E. Li, "Study on distribution and dynamic accumulation of catalpol and total iridoid in fresh Rehmannia glutinosa," China Journal of Chinese Materia Medica, vol. 39, no. 3, pp. 466-470, 2014.

[17] J. J. Wang, Z. L. Zhang, P. P. Du, and Y. F. Sun, "Determination of catalpol and verbascoside in Rheminna glutinosa Libosch leaves and root tubers by UPLC," Chinese Traditional Patent Medicine, no. 9, pp. 1981-1984, 2015.

[18] J. Xu, J. Wu, L.-Y. Zhu et al., "Simultaneous determination of iridoid glycosides, phenethylalcohol glycosides and furfural derivatives in Rehmanniae Radix by high performance liquid chromatography coupled with triple-quadrupole mass spectrometry," Food Chemistry, vol. 135, no. 4, pp. 2277-2286, 2012.

[19] G.-M. Fu, S.-P. Shi, F. C. F. Ip, H.-H. Pang, and N. Y. Ip, "A new carotenoid glycoside from Rehmannia glutinosa," Natural Product Research, vol. 25, no. 13, pp. 1213-1218, 2011.

[20] Y.-M. Ma, J.-H. Guo, C.-W. Tian, and T.-J. Zhang, "Determination of catalpol and aucubin in fresh Rehmannia glutinosa by HPLC," Chinese Traditional and Herbal Drugs, vol. 42, no. 7, pp. 1348-1350, 2011.

[21] H. Li, B. Chen, Z. Zhang, and S. Yao, "Focused microwaveassisted solvent extraction and HPLC determination of effective constituents in Eucommia ulmodies Oliv. (E. ulmodies)," Talanta, vol. 63, no. 3, pp. 659-665, 2004.

[22] H. M. Cui, A. D. Yang, and H. Luo, "Determination of aucubin and catalpol in Vironica Eriogyine H. Winkl from different habitats by HPLC," Modernization of Traditional Chinese Medicine and Materia Medica-World Science and Technology, vol. 16, pp. 1025-1028, 2014.

[23] E. Piątczak, Ł. Kuźma, W. Porada, B. Olas, and H. Wysokińska, "Evaluation of antioxidant properties of methanolic extracts from leaves and roots of Rehmannia glutinosa Libosch. In human blood," Acta Poloniae Pharmaceutica, vol. 72, no. 4, pp. 777-783, 2015.

[24] S. Damtoft, "Biosynthesis of catalpol," Phytochemistry, vol. 35, no. 5, pp. 1187-1189, 1994. 

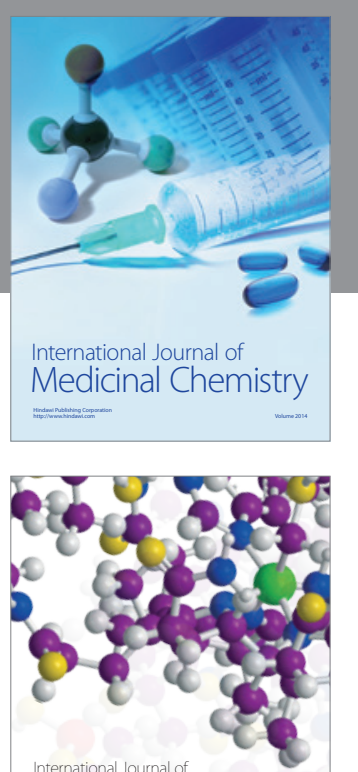

Carbohydrate Chemistry

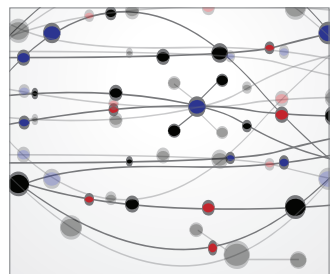

The Scientific World Journal
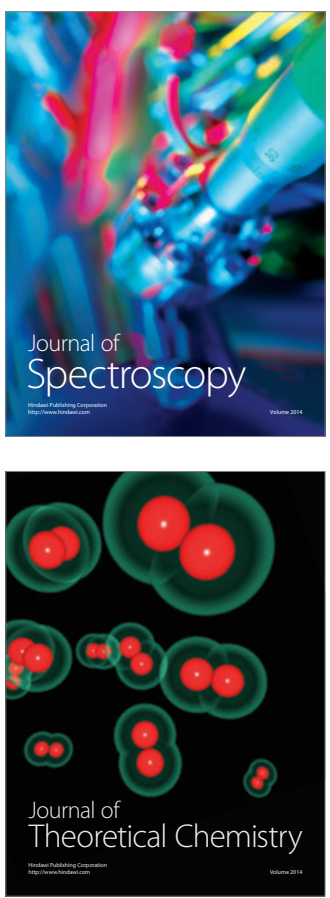
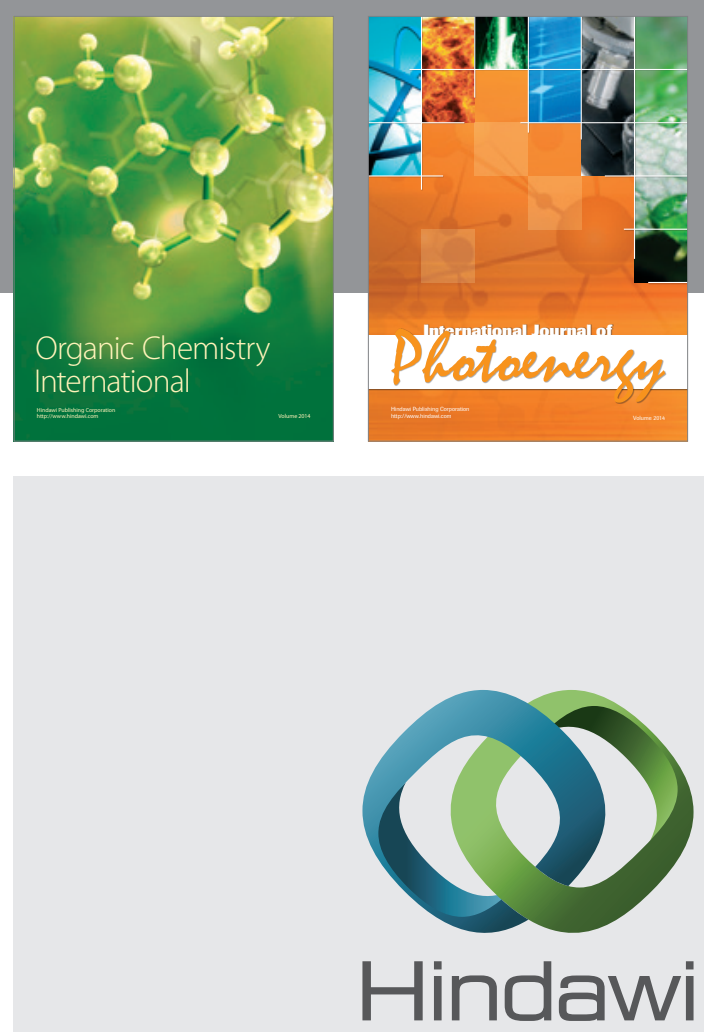

Submit your manuscripts at

http://www.hindawi.com

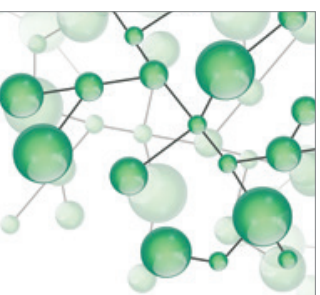

International Journal of

Inorganic Chemistry

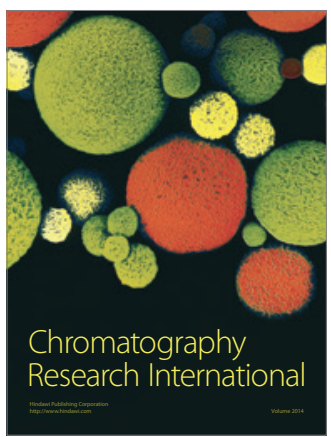

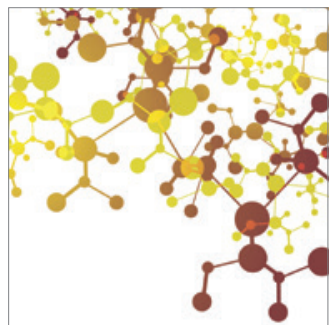

Applied Chemistry
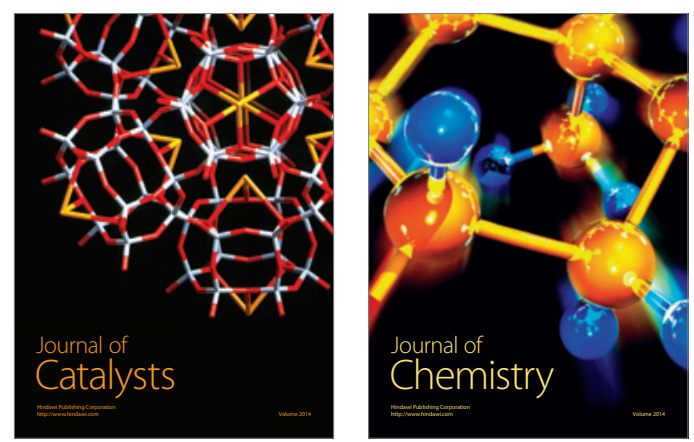
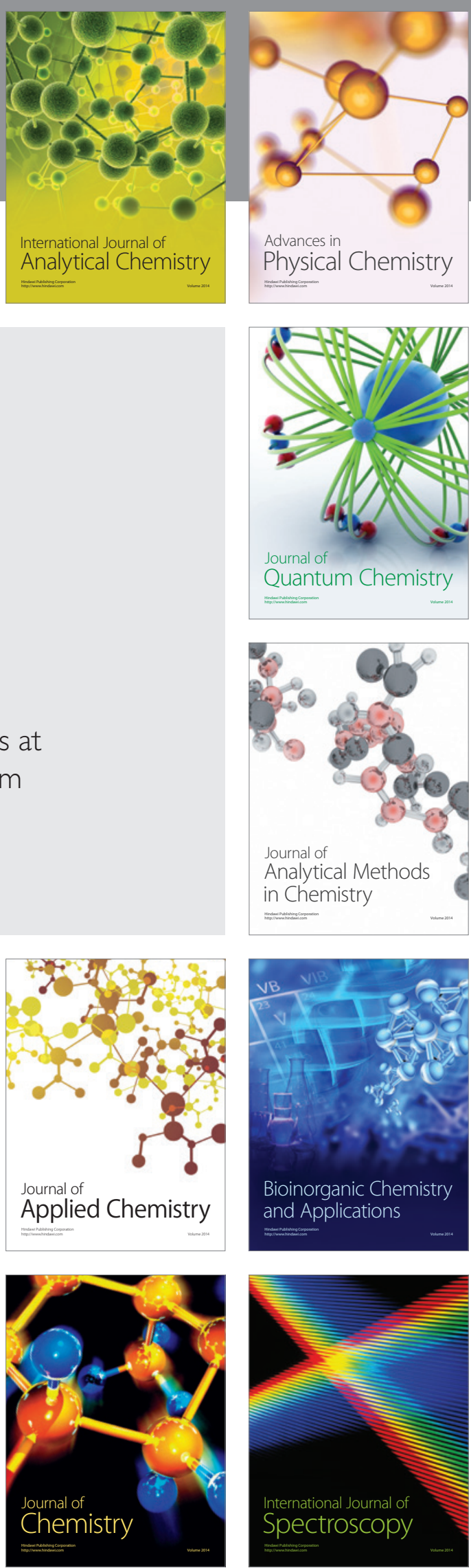